Research Article

\title{
Walking Characteristics of Dual-Arm Inspection Robot with Flexible-Cable
}

\author{
Yongle Wei $\mathbb{D},{ }^{1}$ Jiashun Zhang $\mathbb{D},{ }^{1}$ and Lijin Fang $\mathbb{D}^{2}$ \\ ${ }^{1}$ College of Mechanical Engineering, Liaoning Technical University, Fuxin 123000, China \\ ${ }^{2}$ Faculty of Robot Science and Engineering, Northeastern University, Shenyang 110819, China
}

Correspondence should be addressed to Yongle Wei; weiyongle126@126.com

Received 29 August 2020; Revised 28 February 2021; Accepted 12 April 2021; Published 20 April 2021

Academic Editor: Changsheng Li

Copyright (c) 2021 Yongle Wei et al. This is an open access article distributed under the Creative Commons Attribution License, which permits unrestricted use, distribution, and reproduction in any medium, provided the original work is properly cited.

The overhead transmission line has a catenary shape, which has great influence on the dynamic characteristics of an inspection robot walking along the line and may even cause the walking-wheel to fall from the line. Compared with other similar inspection robots, the unique structure of the dual-arm inspection robot with flexible-cable is introduced. Taking the dual-arm inspection robot with flexible-cable walking along the uphill section of the line as an example, the force states of the robot when it works at acceleration, uniform speed, deceleration, and stopping were studied in detail. The corresponding force balance equations were established, and the walking-wheel torques in each working state were solved. The working states of the robot walking along the catenary shape line were simulated using ADAMS software. Simulation results show that the walking process of the robot is stable, the walking-wheels have good contact with the line, and the forces of two walking-wheels are almost balanced, which enables the robot to have good adaptability and climbing ability for the line. The prototype test that the robot walked along the line was carried out. The results of the simulation and prototype test are consistent with the theoretical analysis, so the rationality of robot structure design is verified. In the future, the navigation control and stability of the robot walking along the line will be researched, so that the robot can complete the patrol task in the real environment.

\section{Introduction}

In order to ensure the safety and stable operation of an overhead high-voltage transmission line and detect its defects, it is necessary to conduct regular inspections for the transmission line. Currently, the main inspection methods include foot patrol and helicopter-assisted ones. However, due to the recent development of the robot technology, using a robot to inspect the transmission line has become one of the research hotspots. Some scholars and research institutes have developed several dual-arm inspection robots.

Palulo et al. presented a robot named "Expliner" $[1,2]$, each arm of the robot had two walking-wheels, and the robot only walked on the dividing-line. Pouliot et al. developed a robot named "LineScout" $[3,4]$, the robot had two arms on which two walking-wheels were installed, respectively, and the robot had a set of separate clamp mechanism which helped the robot cross the obstacles. Alisson et al. reported a robot which has two walking-wheels on its two arms and can be driven by a combustion engine or an electric engine [5]. The common feature of the above mentioned robots is that two arms of each robot are mounted perpendicularly and fixedly on the body, and the length of each arm is unchangeable.

Ed Boje et al. illustrated a robot where two arms construct in series and the lower ends of two arms hinge on the same axis $[6,7]$. Ludan Wang et al. also developed a robot with similar structures $[8,9]$. The common feature of two robots is that two arms of each robot form a triangle with the line and the length of each arm is unchangeable.

Zhu Xinglong et al.[10, 11], WU Gongping et al.[12, 13], and Zhang Guoxian et al. [14, 15] also reported the development of dual-arm robots with similar structures. The common feature of three robots is that two arms of each robot are mounted perpendicularly on its body. Each arm can move along the body, and its length can be changed. 
Consequently, the working postures of this type of robot are flexible and varied.

Although the structures of above three type dual-arm inspection robots are different and the robots work in various ways, they have the same defects that the forces of two arms' walking-wheels are not equal and the walkingwheels skid easily when robot walks along the large slope line [10]. Currently, the arms of the most inspection robots are rigid; however, the load carrying capacity of the cable is very good and it is not used in the inspection robot.

M. H. Korayem et al. built a cable robot and researched the control approach of the robot $[16,17]$. Based on their research results, applying the cable to the inspection robot maybe solves the above problems, and a dual-arm inspection robot with flexible-cable was presented by WEI Yongle et al. $[18,19]$. The research goal is to develop a robot with the strong ability of obstacle-crossing, almost equal forces of two walking-wheels, and simpler posture control [20, 21].

In this paper, the force states of the dual-arm inspection robot with flexible-cable were studied in detail when it walked along the line. The forces and torques of two walking-wheels were simulated and the prototype test was carried out.

In order to inspect the line automatically in the real environment $[22,23]$, the navigation control and stability of the robot walking along the line must be studied in depth, and it will be the focused research in the future.

Pavol Božek et al. studied the navigation control and stability of a mobile robot. They formulated the dynamics model of the mobile robot using Newton-Euler method [24], and used the inertial navigation to control the mobile platform [25]. Their research will provide reference for our next work.

\section{Structure Characteristics of the Dual-Arm Inspection Robot with Flexible-Cable}

The overhead high-voltage transmission line is spanned on the power iron tower through the clamps and insulator sets, etc., and the line has a catenary shape. There are many obstacles near the suspension point of the line, such as clamp, insulator set, damper, etc. Therefore, the robot must have the strong climbing ability and the obstacle-crossing ability when it walks along the line.

2.1. Structure of the Robot. According to the working environment of the robot, the dual-arm inspection robot with flexible-cable was proposed in [18] and the obstacle-crossing process of the robot was introduced in detail, but its walking process was not researched.

The arm of the robot adapts a humanoid structure, and each arm has four degrees of freedom. The walking-wheel and the clamping mechanism are installed at the end of the arm. A variable-length flexible-cable is installed between the wrist joint and the mobile platform, and the mobile platform can move along the box body with one end of the flexiblecable. The structure of the robot is shown in Figure 1.

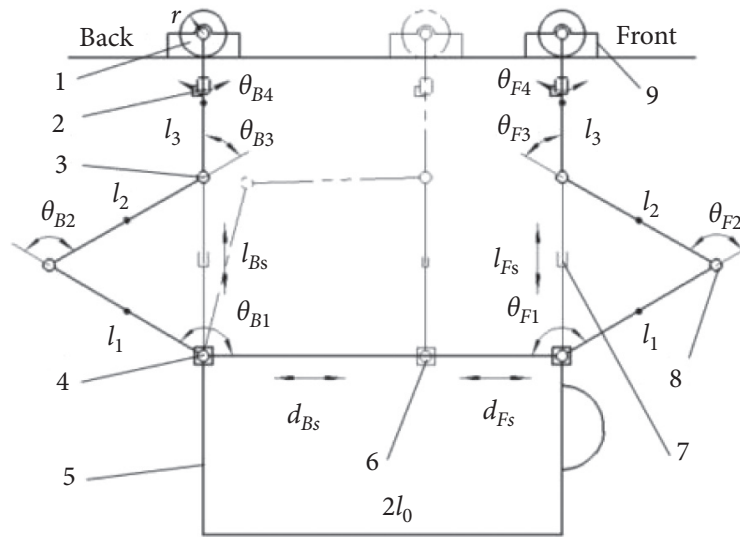

$\begin{array}{lll}1 \text { - Walking-wheel } & 4 \text { - Shoulder joint } & 7 \text { - Flexible-cable } \\ 2 \text { - Rotary joint } & 5 \text { - Box body } & 8 \text { - Elbow joint } \\ 3 \text { - Wrist joint } & 6 \text { - Mobile platform } & 9 \text { - Clamping mechanism }\end{array}$

Figure 1: Robot structure.

The robot can walk along the line by two walking-wheels rolling; meanwhile, the joints of two arms are relaxed and the lengths of two flexible-cables remain unchanged.

The robot can cross various obstacles of the line by changing the lengths of two flexible-cables and adjusting the joints angles of two arms and moving the mobile platforms along the box body.

In Figure $1, r$ is the radius of the walking-wheel, $l_{1}$ and $l_{2}$ and $l_{3}$ are the length of each section of the arm, $2 l_{0}$ is the length of the box body, $\theta_{1}$ and $\theta_{2}$ and $\theta_{3}$ are the angle of each joint of the arm, $l_{s}$ is the length of the flexible-cable, and $d_{s}$ is the moving distance of the mobile platform. All the structure parameters and motion parameters of the robot are shown in Table 1 .

2.2. Structure Characteristics of the Robot. Because the line slope is constantly changing, the structures of the inspection robots proposed by some previous researchers have obvious deficiencies at the line adaptation [1-15]. For example, when these robots walk along the line with gradually increasing slope, as shown in Figure 2, the forces of two walking-wheels are quite different because of the robot posture unchanging, which will make the walking-wheel's force little to skid easily and even fall from the line [21].

Figure 2 shows that when the robot that its arms are perpendicular to the box body walks along the uphill section, with the center of gravity of the robot moving forward, the force of the front arm's walking-wheel $F_{F}$ is obviously larger than the force of the back arm's walking-wheel $F_{B}$, and even the $F_{B}$ may be equal to zero.

However, when the dual-arm inspection robot with flexiblecable walks along the line, each joint of the arm is relaxed and the forces of two walking-wheels are about equal because of the robot posture changing, so the walking-wheels do not easily skid, which makes the robot adapt to the line slope very well, as shown in Figure 3.

Figure 3 shows that because the robot posture can be changed with the line, the center of gravity of the robot is always between two walking-wheels, so $F_{F}$ and $F_{B}$ can be about equal. 
TABLE 1: Structure and motion parameters of the robot.

\begin{tabular}{lccc}
\hline $\begin{array}{l}\text { Structure } \\
\text { parameters }\end{array}$ & $\begin{array}{c}\text { Value } \\
(\mathrm{mm})\end{array}$ & $\begin{array}{c}\text { Motion } \\
\text { parameters }\end{array}$ & Range \\
\hline$l_{0}$ & 325 & $\theta_{B 1}, \theta_{F 1}$ & $20^{\circ}$ to $190^{\circ}$ \\
$l_{1}$ & 300 & $\theta_{B 2}, \theta_{F 2}$ & $0^{\circ}$ to $170^{\circ}$ \\
$l_{2}$ & 300 & $\theta_{B 3}, \theta_{F 3}$ & $-120^{\circ}$ to $120^{\circ}$ \\
$l_{3}$ & 270 & $\theta_{B 4}, \theta_{F 4}$ & $-180^{\circ}$ to \\
& & & $180^{\circ}$ \\
$r$ & 40 & $l_{B s}, l_{F s}$ & 50 to \\
& & $d_{B s}, d_{F s}$ & $050 \mathrm{~mm}$ \\
& & & \\
\hline
\end{tabular}

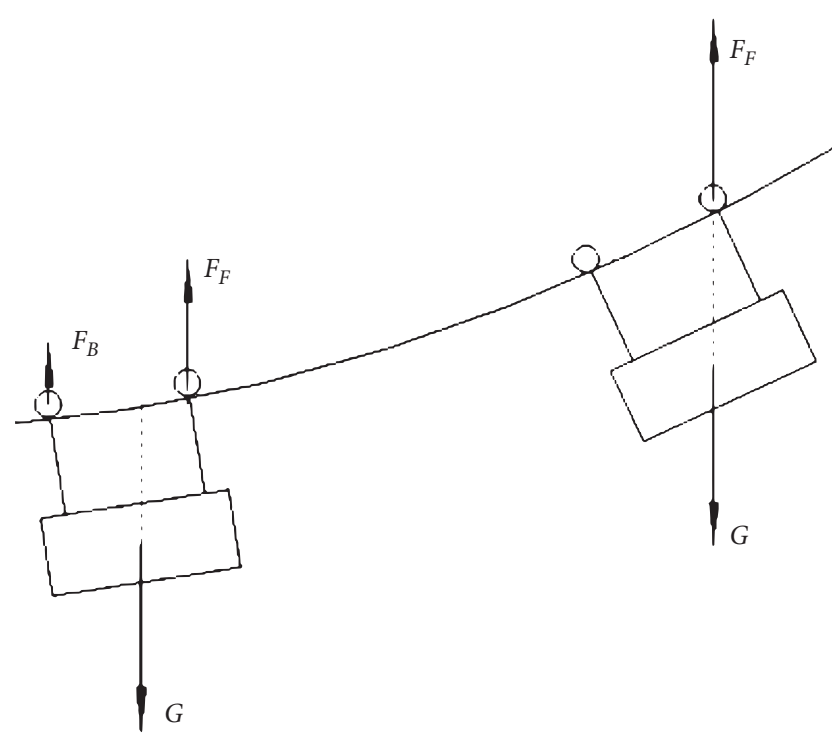

FIGURE 2: Walking-wheels' forces of robot where its arms are perpendicular to the box body.

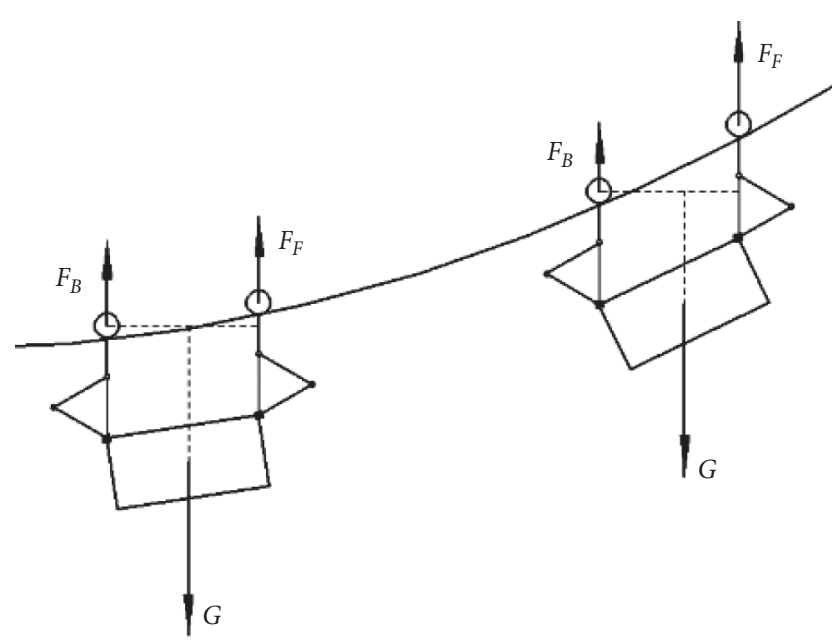

FIGURE 3: Walking-wheels' forces of the dual-arm. Inspection robot with flexible-cable.

From Figures 2 and 3, it can be seen intuitively that the walking-posture of the dual-arm inspection robot with flexible-cable is noticeably different with the walking- posture of the other inspection robot. So the walking dynamic characteristics of this robot are unique and they need to be researched in detail, which will provide the theoretical basis for the manufacture and motion control of the robot prototype in future.

\section{Theoretical Analysis on Walking Dynamic Characteristics of the Robot}

When the robot walks along the catenary line, there will be two cases of uphill and downhill. The working states of robot have four states: acceleration, uniform speed, deceleration, and stopping, and the walking-wheel needs to provide driving or braking torque. Because the curvature radius formed by the line drape is much larger than the distance of the two walking-wheels, it can be considered that the line between the two walking-wheels is a straight line. Taking the robot walking along the uphill section as an example, the walking dynamic characteristics of the robot at various working states are analyzed, and it is similar that the robot walks along the downhill section, so it is not repeated.

3.1. Stop State of the Robot. When the robot stops on the line, the friction torques of two walking-wheels' shafts are so small that the walking-wheel will roll down along the line. In order to keep the robot stopping, it is necessary for two walking-wheels to provide the braking torque.

3.1.1. Force Analysis of the Robot. When the robot remains static on the line, its posture and force state are shown in Figure 4.

According to Figure 4(a), the geometric relationship of the robot at stop state and the force balance equations of two walking-wheels' shafts are established as follows:

$$
\left\{\begin{array}{l}
\overline{O_{B 1} G}=\sqrt{l_{0}^{2}+h^{2}}, \\
\overline{O_{B 4} C}=\overline{O_{B 1} H}=\overline{O_{B 1} G} \cos (\theta-\alpha), \\
\overline{O_{B 4} D}=2 l_{0} \cos \alpha \\
\overline{O_{B 4} E}=\overline{D F} \\
\theta=\arctan \left(\frac{h}{l_{0}}\right)
\end{array}\right.
$$

where $l_{0}$ is half of the length of the box body, $h$ is the distance between the gravity of the robot and the top surface of the box body, and $\alpha$ is the transmission line slope. The same parameters of subsequent formulas are the same means.

$$
\left\{\begin{array}{l}
F_{F}+F_{B}=m_{0} g+2\left(m_{1}+m_{2}+m_{3}+m_{5}\right) g, \\
F_{F} \overline{O_{B 4} D}-m_{3} g \overline{O_{B 4} D}-\left(m_{1}+m_{2}\right) g\left(\overline{O_{B 4} D}+\overline{D F}\right), \\
-m_{5} g \overline{O_{B 4} D}-m_{0} g \overline{O_{B 4} C}+\left(m_{1}+m_{2}\right) g \overline{O_{B 4} E}=0,
\end{array}\right.
$$




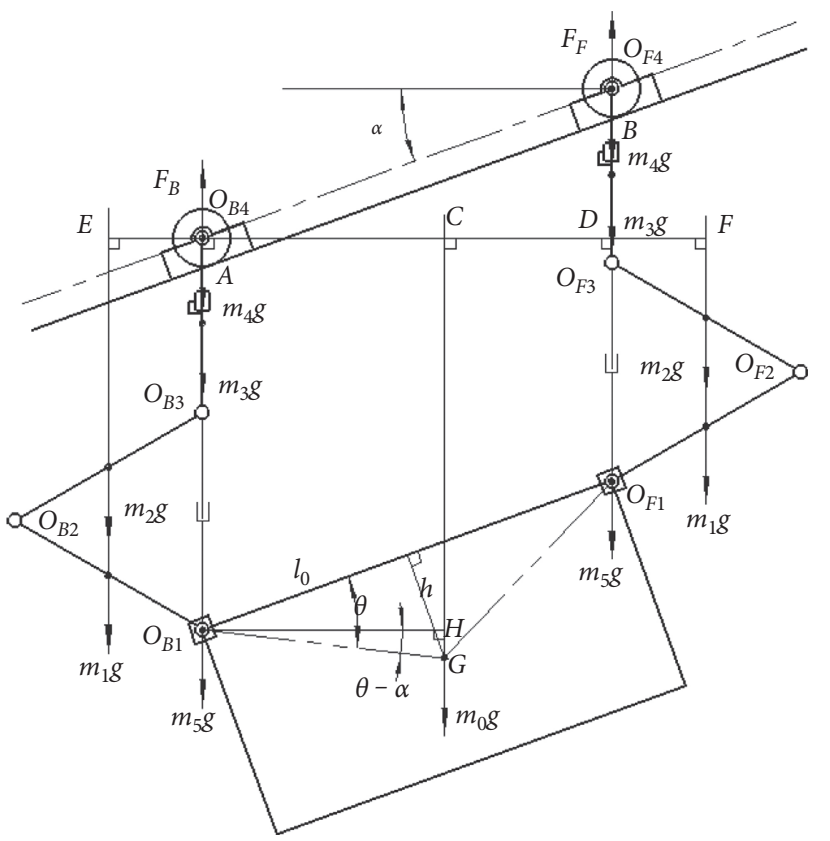

(a)

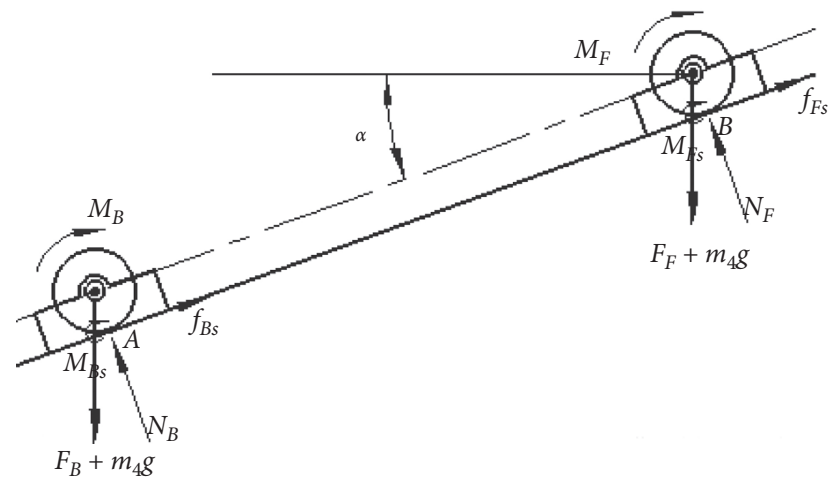

(b)

FIGURE 4: Force analysis of robot when it stops. (a) Force state of robot. (b) Force state of walking-wheels.

where $F_{F}$ is the force of the front arm's walking-wheel, $F_{B}$ is the force of the back arm's walking-wheel, $m_{0}$ is the mass of the box, $m_{1}, m_{2}, m_{3}$ is the mass of each section of each arm, respectively, $m_{4}$ is the mass of the walking-wheel, $m_{5}$ is the mass of the mobile platform, and $g$ is the acceleration of gravity. The same parameters of subsequent formulas are the same means.

The forces of two walking-wheels are solved by formulas (1) and (2) to

$$
\left\{\begin{array}{l}
F_{F}=\left(m_{1}+m_{2}+m_{3}+m_{5}\right) g+m_{0} g \frac{\sqrt{l_{0}^{2}+h^{2}} \cos (\theta-\alpha)}{2 l_{0} \cos \alpha}, \\
F_{B}=\left(m_{1}+m_{2}+m_{3}+m_{5}\right) g+m_{0} g\left(1-\frac{\sqrt{l_{0}^{2}+h^{2}} \cos (\theta-\alpha)}{2 l_{0} \cos \alpha}\right) .
\end{array}\right.
$$

From formula (3), it can be seen that the forces of two walking-wheels are related to the gravity of the robot and the line slope. Because $\alpha$ is greater than zero, $\sqrt{l_{0}^{2}+h^{2}} \cos (\theta-\alpha)$ is greater than $l_{0}$ and $l_{0} \cos \alpha$ is less than $l_{0}$, and there is

$$
\frac{\sqrt{l_{0}^{2}+h^{2}} \cos (\theta-\alpha)}{2 l_{0} \cos \alpha}>\frac{1}{2} \text {. }
$$

So, $F_{F}$ is greater than $F_{B}$, and as $\alpha$ increases, $F_{F}$ increases and $F_{B}$ decreases.

3.1.2. Torques of Two Walking-Wheels. When the robot stops on the line, in order to avoid skidding the walkingwheel must provide the upward driving torque. According to the force state shown in Figure 4(b), it can be seen that the torques of two walking-wheels are

$$
\left\{\begin{array}{l}
N_{F}=\left(F_{F}+m_{4} g\right) \cos \alpha, \\
M_{F \delta}=N_{F} \delta, \\
f_{F}=\left(F_{F}+m_{4} g\right) \sin \alpha, \\
f_{F} r-M_{F \delta} \leq M_{F} \leq f_{F} r+M_{F \delta}, \\
N_{B}=\left(F_{B}+m_{4} g\right) \cos \alpha, \\
M_{B \delta}=N_{B} \delta, \\
f_{B}=\left(F_{B}+m_{4} g\right) \sin \alpha, \\
f_{B} r-M_{B \delta} \leq M_{B} \leq f_{B} r+M_{B \delta},
\end{array}\right.
$$

where $N_{F}$ is the normal force of the line to the front arm's walking-wheel, $f_{F}$ is the friction of the line to the front arm's walking-wheel, $M_{F \delta}$ is the rolling friction couple moment of the line to the front arm's walking-wheel, $M_{F}$ is the torque of the front arm's walking-wheel, $N_{B}$ is the normal force of the line to the back arm's walking-wheel, $f_{B}$ is the friction of the 
line to the back arm's walking-wheel, $M_{B \delta}$ is the rolling friction couple moment of the line to the back arm's walking-wheel, $M_{B}$ is the torque of the back arm's walkingwheel, $\delta$ is the rolling friction coefficient of the line to the walking-wheel, and $r$ is the radius of the walking-wheel. The same parameters of subsequent formulas are the same means.

The torques of two walking-wheels are solved by formulas (3) and (5) to

$$
\left\{\begin{array}{l}
M_{F} \geq\left(F_{F}+m_{4} g\right)(r \sin \alpha-\delta \cos \alpha), \\
M_{F} \leq\left(F_{F}+m_{4} g\right)(r \sin \alpha+\delta \cos \alpha), \\
M_{B} \geq\left(F_{B}+m_{4} g\right)(r \sin \alpha-\delta \cos \alpha), \\
M_{B} \leq\left(F_{B}+m_{4} g\right)(r \sin \alpha+\delta \cos \alpha) .
\end{array}\right.
$$

According to formula (3), $F_{F}$ is greater than $F_{B}$, so $M_{F}$ is greater than $M_{B}$. However, according to formula (6), $M_{F}$ and $M_{B}$ are mainly affected by $\alpha$ besides $F_{F}$ and $F_{B}$.

3.2. Uniform Speed Walking of the Robot. When the robot walks at a constant speed, its force state is the same as the force state of the robot stopping which is shown in Figure 4.

The walking-wheel must provide an upward driving torque. According to the force state shown in Figure 4(b), it can be seen that the torques of two walking-wheels are

$$
\left\{\begin{array}{l}
N_{F}=\left(F_{F}+m_{4} g\right) \cos \alpha, \\
M_{F \delta}=N_{F} \delta, \\
f_{F}=\left(F_{F}+m_{4} g\right) \sin \alpha, \\
M_{F}-M_{F \delta}-f_{F} r=0, \\
N_{B}=\left(F_{B}+m_{4} g\right) \cos \alpha, \\
M_{B \delta}=N_{B} \delta, \\
f_{B}=\left(F_{B}+m_{4} g\right) \sin \alpha, \\
M_{B}-M_{B \delta}-f_{B} r=0 .
\end{array}\right.
$$

The torques of two walking-wheels are solved by formulas (3) and (7) to

$$
\left\{\begin{array}{l}
M_{F}=\left(F_{F}+m_{4} g\right)(r \sin \alpha+\delta \cos \alpha), \\
M_{B}=\left(F_{B}+m_{4} g\right)(r \sin \alpha+\delta \cos \alpha) .
\end{array}\right.
$$

It is similar to two walking-wheels' torques of the robot stopping that $M_{F}$ is greater than $M_{B}$ because $F_{F}$ is greater than $F_{B}$.
3.3. Accelerated Walking of the Robot. When the robot accelerates, it will tilt backward at an angle due to inertia, as shown in Figure 5(a).

3.3.1. Force Analysis of the Robot. The robot force state is shown in Figure 5 when it accelerates.

According to Figure 5(a), the geometric relationship of the robot accelerating and the force balance equations of two walking-wheels' shafts are established as shown in formulas (9) and (10):

$$
\left\{\begin{array}{l}
\overline{O_{B 1} G}=\sqrt{l_{0}^{2}+h^{2}}, \\
\overline{O_{B 4} C}=\overline{O_{B 1} H}=\overline{O_{B 1} G} \cos \phi, \\
\overline{O_{B 4} D}=2 l_{0} \cos (\alpha+\beta), \\
\overline{O_{B 4} E}=\overline{D F}, \\
\theta=\arctan \left(\frac{h}{l_{0}}\right), \\
\beta=\arcsin \left[\left(\frac{a}{g_{e}}\right) \sin \left(\frac{\pi}{2+\alpha}\right)\right], \\
g_{e}=\sqrt{a^{2}+g^{2}-2 a g \cos \left(\frac{\pi}{2+\alpha}\right)}, \\
\phi=\theta-\alpha-\beta,
\end{array}\right.
$$

where $\beta$ is the angle at which the robot tilts due to inertia and $a$ is the acceleration of robot. The same parameters of subsequent formulas are the same means.

$$
\left\{\begin{array}{l}
F_{F}+F_{B}=m_{0} g_{e}+2\left(m_{1}+m_{2}+m_{3}+m_{5}\right) g_{e}, \\
F_{F} \overline{O_{B 4} D}-m_{3} g_{e} \overline{O_{B 4} D}-\left(m_{1}+m_{2}\right) g_{e}\left(\overline{O_{B 4} D}+\overline{D F}\right), \\
-m_{5} g_{e} \overline{O_{B 4} D}-m_{0} g_{e} \overline{O_{B 4} C}+\left(m_{1}+m_{2}\right) g_{e} \overline{O_{B 4} E}=0 .
\end{array}\right.
$$

The forces of two walking-wheels are solved by formulas (9) and (10) to

$$
\left\{\begin{array}{l}
F_{F}=\left(m_{1}+m_{2}+m_{3}+m_{5}\right) g_{e}+m_{0} g_{e} \frac{\sqrt{l_{0}^{2}+h^{2}} \cos (\theta-\alpha-\beta)}{2 l_{0} \cos (\alpha+\beta)} \\
F_{B}=\left(m_{1}+m_{2}+m_{3}+m_{5}\right) g_{e}+m_{0} g_{e}\left(1-\frac{\sqrt{l_{0}^{2}+h^{2}} \cos (\theta-\alpha-\beta)}{2 l_{0} \cos (\alpha+\beta)}\right) .
\end{array}\right.
$$




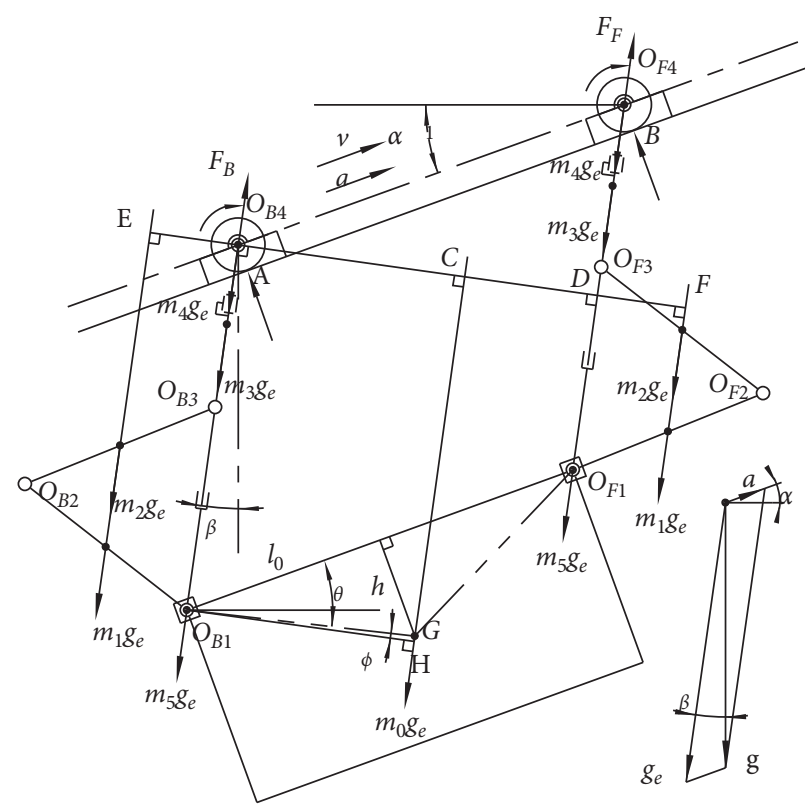

(a)

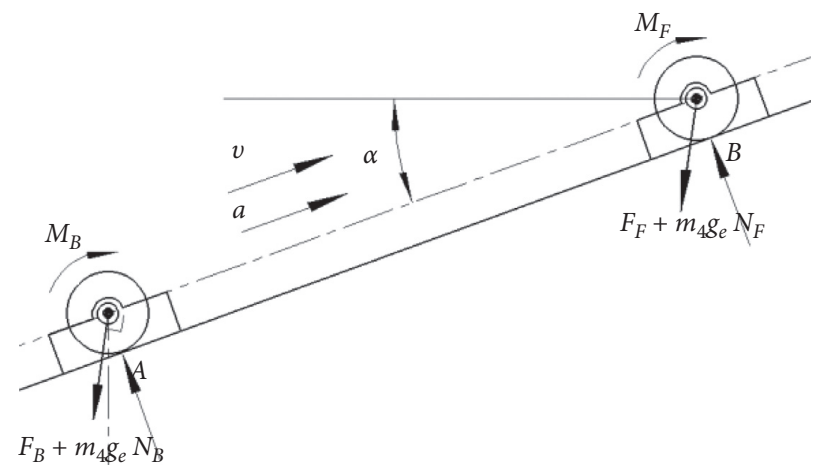

FIgURE 5: Force analysis of robot when it accelerates. (a) Force state of robot. (b) Force state of walking-wheels.

From formula (11), it can be seen that the forces of two walking-wheels are related to acceleration of the robot besides the gravity of the robot and the line slope. Because $(\alpha+\beta)$ is greater than zero, $\sqrt{l_{0}^{2}+h^{2}} \cos (\theta-\alpha-\beta)$ is greater than $l_{0}$ and $l_{0} \cos (\alpha+\beta)$ is less than $l_{0}$, and there is

$$
\frac{\sqrt{l_{0}^{2}+h^{2}} \cos (\theta-\alpha-\beta)}{2 l_{0} \cos (\alpha+\beta)}>\frac{1}{2} \text {. }
$$

So, $F_{F}$ is greater than $F_{B}$, and $a$ is greater, $\beta$ is greater correspondingly, and the difference between $F_{F}$ and $F_{B}$ goes up also.

3.3.2. Torques of Two Walking-Wheels. When the robot accelerates along the line, the walking-wheels must provide the larger upward driving torque. According to the force state as shown in Figure 5(b), it can be seen that the torques of two walking-wheels are

$$
\left\{\begin{array}{l}
N_{F}=\left(F_{F}+m_{4} g_{e}\right) \cos (\alpha+\beta), \\
M_{F \delta}=N_{F} \delta, \\
f_{F}=\left(F_{F}+m_{4} g_{e}\right) \sin (\alpha+\beta), \\
M_{F}-M_{F \delta}-f_{F} r=0, \\
N_{B}=\left(F_{B}+m_{4} g_{e}\right) \cos (\alpha+\beta), \\
M_{B \delta}=N_{B} \delta \\
f_{B}=\left(F_{B}+m_{4} g_{e}\right) \sin (\alpha+\beta), \\
M_{B}-M_{B \delta}-f_{B} r=0 .
\end{array}\right.
$$

The torques of two walking-wheels are solved by formulas (11) and (13) to

$$
\left\{\begin{array}{l}
M_{F}=\left(F_{F}+m_{4} g_{e}\right)[r \sin (\alpha+\beta)+\delta \cos (\alpha+\beta)], \\
M_{B}=\left(F_{B}+m_{4} g_{e}\right)[r \sin (\alpha+\beta)+\delta \cos (\alpha+\beta)] .
\end{array}\right.
$$

So, $M_{F}$ and $M_{B}$ are mainly affected by $\alpha$ and $\beta$ besides $F_{F}$ and $F_{B}$.
3.4. Decelerated Walking of the Robot. When the robot decelerates, it will tilt forward at an angle due to inertia, as shown in Figure 6(a).

3.4.1. Force Analysis of the Robot. The robot force state is shown in Figure 6 when it decelerates. 


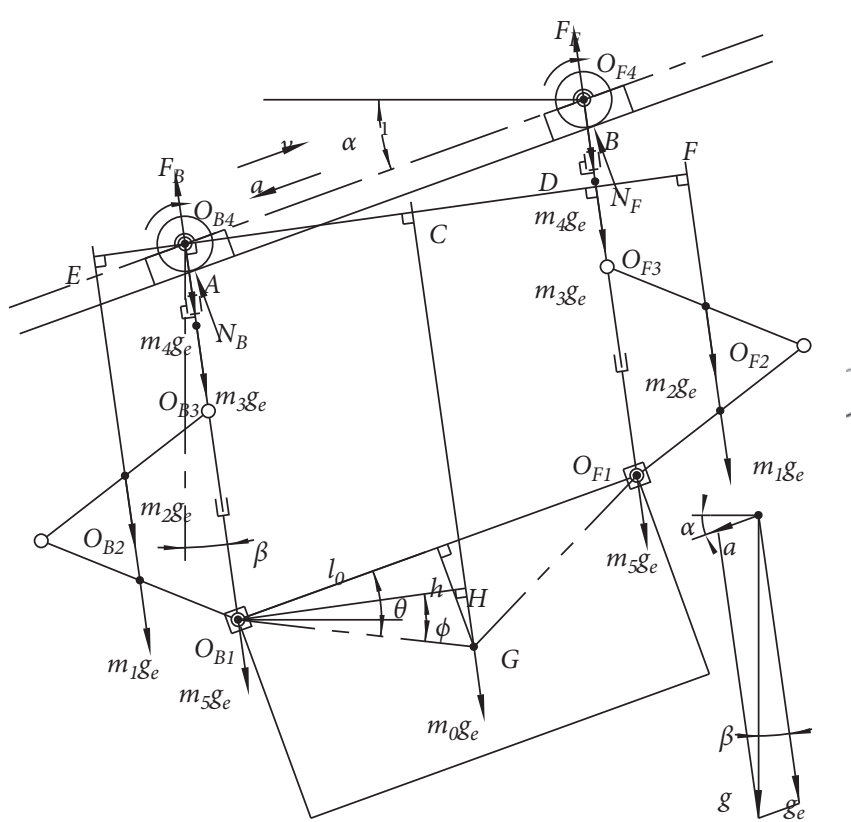

(a)

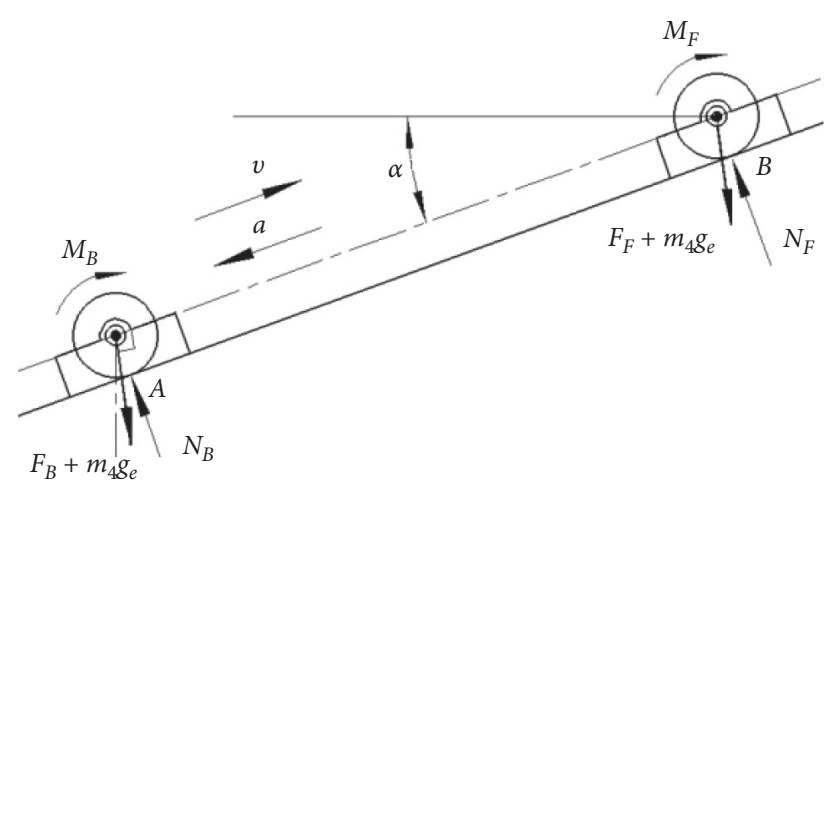

(b)

FIGURE 6: Force analysis of robot when it decelerates. (a) Force state of robot. (b) Force state of walking-wheels.

According to Figure 6(a), the geometric relationship of the robot decelerating and the force balance equations of two walking-wheels' shafts are established as shown in formulas (15) and (16):

$$
\left\{\begin{array}{l}
\overline{O_{B 1} G}=\sqrt{l_{0}^{2}+h^{2}}, \\
\overline{O_{B 4} C}=\overline{O_{B 1} H}=\overline{O_{B 1} G} \cos \phi \\
\overline{O_{B 4} D}=2 l_{0} \cos (\alpha-\beta), \\
\overline{O_{B 4} E}=\overline{D F} \\
\theta=\arctan \left(\frac{h}{l_{0}}\right) \\
\beta=\arcsin \left[\left(\frac{a}{g_{e}} \sin \left(\frac{\pi}{2-\alpha}\right)\right)\right] \\
g_{e}=\sqrt{a^{2}+g^{2}-2 a g \cos \left(\frac{\pi}{2-\alpha}\right)} \\
\phi=\theta-\alpha+\beta \\
F_{F}+F_{B}=m_{0} g_{e}+2\left(m_{1}+m_{2}+m_{3}+m_{5}\right) g_{e} \\
-m_{5} g_{e} \overline{O_{B 4} D}-m_{0} g_{e} \overline{O_{B 4} C}+\left(m_{1}+m_{2}\right) g_{e} \overline{O_{B 4} E}=0
\end{array}\right.
$$

The forces of two walking-wheels are solved by formulas (15) and (16) to 


$$
\left\{\begin{array}{l}
F_{F}=\left(m_{1}+m_{2}+m_{3}+m_{5}\right) g_{e}+m_{0} g_{e} \frac{\sqrt{l_{0}^{2}+h^{2}} \cos (\theta-\alpha+\beta)}{2 l_{0} \cos (\alpha-\beta)} \\
F_{B}=\left(m_{1}+m_{2}+m_{3}+m_{5}\right) g_{e}+m_{0} g_{e}\left(1-\frac{\sqrt{l_{0}^{2}+h^{2}} \cos (\theta-\alpha+\beta)}{2 l_{0} \cos (\alpha-\beta)}\right) .
\end{array}\right.
$$

In formula (17), if $(\alpha-\beta)$ is greater than zero, $\sqrt{l_{0}^{2}+h^{2}} \cos (\theta-\alpha+\beta)$ is greater than $l_{0}$ and $l_{0} \cos (\alpha-\beta)$ is less than $l_{0}$, and there is

$$
\frac{\sqrt{l_{0}^{2}+h^{2}} \cos (\theta-\alpha+\beta)}{2 l_{0} \cos (\alpha-\beta)}>\frac{1}{2} .
$$

If $(\alpha-\beta)$ is zero, $\sqrt{l_{0}^{2}+h^{2}} \cos (\theta-\alpha+\beta)$ is equal to $l_{0}$ and $l_{0} \cos (\alpha-\beta)$ is equal to $l_{0}$ too, and there is

$$
\frac{\sqrt{l_{0}^{2}+h^{2}} \cos (\theta-\alpha+\beta)}{2 l_{0} \cos (\alpha-\beta)}=\frac{\sqrt{l_{0}^{2}+h^{2}} \cos (\theta)}{2 l_{0} \cos \left(0^{\circ}\right)}=\frac{1}{2} \text {. }
$$

If $(\alpha-\beta)$ is less than zero, $\sqrt{l_{0}^{2}+h^{2}} \cos (\theta-\alpha+\beta)$ is less than $l_{0}$ and $l_{0} \cos (\alpha-\beta)$ is less than $l_{0}$ too. Because $\theta$ is much bigger than zero, according to the law of cosine function, there is

$$
\begin{gathered}
\frac{\cos (\theta-\alpha+\beta)}{\cos (\alpha-\beta)}=\frac{\cos [\theta-(\alpha-\beta)]}{\cos [0+(\alpha-\beta)]}<\frac{\cos (\theta)}{\cos \left(0^{\circ}\right)}, \\
\frac{\sqrt{l_{0}^{2}+h^{2}} \cos (\theta-\alpha+\beta)}{2 l_{0} \cos (\alpha-\beta)}<\frac{\sqrt{l_{0}^{2}+h^{2}} \cos (\theta)}{2 l_{0} \cos \left(0^{\circ}\right)}=\frac{1}{2}, \\
\begin{cases}F_{F}>F_{B}, & (\alpha-\beta>0), \\
F_{F}=F_{B}, & (\alpha-\beta=0), \\
F_{F}<F_{B}, & (\alpha-\beta<0) .\end{cases}
\end{gathered}
$$

Therefore, the forces of two walking-wheels depend on the line slope and the acceleration of the robot. While $\alpha$ is unchanged, $a$ is greater, $\beta$ is greater correspondingly, and $F_{F}$ decreases and $F_{B}$ increases.

3.4.2. Torques of Two Walking-Wheels. When the robot decelerates along the line, two walking-wheels must provide the less upward driving torque. According to the force state as shown in Figure 6(b), it can be seen that the torques of two walking-wheels are

$$
\left\{\begin{array}{l}
N_{F}=\left(F_{F}+m_{4} g_{e}\right) \cos (\alpha-\beta) \\
M_{F \delta}=N_{F} \delta \\
f_{F}=\left(F_{F}+m_{4} g_{e}\right) \sin (\alpha-\beta) \\
M_{F}-M_{F \delta}-f_{F} r=0 \\
N_{B}=\left(F_{B}+m_{4} g_{e}\right) \cos (\alpha-\beta) \\
M_{B \delta}=N_{B} \delta \\
f_{B}=\left(F_{B}+m_{4} g_{e}\right) \sin (\alpha-\beta) \\
M_{B}-M_{B \delta}-f_{B} r=0
\end{array}\right.
$$

The torques of two walking-wheels are solved by formulas (17) and (18) to

$$
\left\{\begin{array}{l}
M_{F}=\left(F_{F}+m_{4} g_{e}\right)[r \sin (\alpha-\beta)+\delta \cos (\alpha-\beta)], \\
M_{B}=\left(F_{B}+m_{4} g_{e}\right)[r \sin (\alpha-\beta)+\delta \cos (\alpha-\beta)] .
\end{array}\right.
$$

According to formula (17), $M_{F}$ and $M_{B}$ will be

$$
\left\{\begin{array}{l}
M_{F}>M_{B},(\alpha-\beta>0) \\
M_{F}=M_{B},(\alpha-\beta=0) \\
M_{F}<M_{B},(\alpha-\beta<0)
\end{array}\right.
$$

From formulas (3), (6), (8), (11), (14), (17), and (23), it can be seen that $F_{F}, F_{B}, M_{F}$, and $M_{B}$ are affected by the line slop $\alpha$ and the acceleration of the robot $a$ besides the gravity of the robot. Because the gravity of the robot is a constant and the acceleration of the robot $a$ is generally small, the line slop $\alpha$ is the main affecting factor.

\section{Simulation Experiment of the Robot Walking along the Transmission Line}

Taking the transmission line that the heights of both suspension points are equal as an example, according to the calculation formula of catenary, the model of the line which is 100 meters long is established and the maximum line slope is $5^{\circ}$. The three-dimensional model of the robot which is about $38 \mathrm{~kg}$ and the transmission line are loaded into ADAMS, and the simulation experiment is carried out.

4.1. Driving Function of the Walking-Wheel. In order to simulate the uniform speed, acceleration, deceleration, and stopping states of the robot walking along the line, the driving function of the walking-wheel constructed by STEP function is as follows.

$720 \mathrm{~d}^{*}$ step (time, $\left.0,0,2.5,-1\right)+720 \mathrm{~d}^{*}$ step (time, $\left.2.5,0,5,1\right) / /$ robot position adjustment stage. $+1440 \mathrm{~d}^{*}$ step (time,10,0,15,1) $+1440 \mathrm{~d}^{*}$ step (time,30,0,35,1)//robot downhill walking stage. $+1440 \mathrm{~d}^{*}$ step (time,40,0,45,-1)+1440d* $\mathrm{d}^{*}$ step (time,125,0,130,1)// robot transitional walking stage. $+1440 \mathrm{~d}^{*}$ step (time,135,0,140,1) $+1440 \mathrm{~d}^{*}$ step (time,153,0,158,1)//robot uphill walking stage.

In the robot position adjustment stage from $0 \mathrm{~s}$ to $5 \mathrm{~s}$, the robot walks to the starting point of the catenary line, which does not belong to the normal inspection walking of the robot. In the robot downhill walking stage from $10 \mathrm{~s}$ to $35 \mathrm{~s}$, the mechanical characteristics of the robot downhill walking are tested. In the robot transitional walking stage from $40 \mathrm{~s}$ to $130 \mathrm{~s}$, the robot walks from the downhill section to the uphill 
section. In the robot uphill walking stage from $135 \mathrm{~s}$ to $158 \mathrm{~s}$, the mechanical characteristics of the robot uphill walking are tested. The rest of the time, the robot stops on the line.

4.2. Simulation Curves of the Robot Walking States. In the simulation experiments, the velocity and acceleration of the box body as well as the forces and torques of two walkingwheels are mainly studied when the robot walks along the line.

4.2.1. Velocity and Displacement Curves of the Robot. Figure 7 shows the velocity and displacement curves of the center of mass of the robot's box body. When the robot walks along the catenary line as acceleration, uniform speed, or deceleration, the velocity curve (S_V_Box) is smooth, which indicates that the robot walks stably and two walking-wheels do not skid. From the displacement curve (S_DM_Box), it can be seen that the robot walks to the lowest point of the catenary line at the $85^{\text {th }}$ second.

4.2.2. Acceleration Curve of the Robot. Figure 8 shows the acceleration curve of the center of mass of the robot's box body.

$10 \mathrm{~s}$ to $15 \mathrm{~s}, 40 \mathrm{~s}$ to $45 \mathrm{~s}$, and $135 \mathrm{~s}$ to $140 \mathrm{~s}$ are the acceleration processes of the robot. $15 \mathrm{~s}$ to $30 \mathrm{~s}, 45 \mathrm{~s}$ to $125 \mathrm{~s}$, and $140 \mathrm{~s}$ to $153 \mathrm{~s}$ are the uniform speed processes of the robot. $30 \mathrm{~s}$ to $35 \mathrm{~s}, 125 \mathrm{~s}$ to $130 \mathrm{~s}$, and $153 \mathrm{~s}$ to $158 \mathrm{~s}$ are the deceleration processes of the robot. The maximum value is about $0.26 \mathrm{~m} / \mathrm{s}^{2}$ when the robot accelerates or decelerates.

When the robot walks as the uniform speed, the acceleration curve fluctuates slightly near $0 \mathrm{~m} / \mathrm{s}^{2}$, which is mainly due to the fact that the trajectory of the box is a curve; the acceleration in the $x$-direction and $y$-direction is changing.

4.2.3. Force Curves of Two Walking-Wheels. Figure 9 shows the force curves between two walking-wheels and the line.

The force of the back arm's walking-wheel (S_F_HB_GunLun) is larger than the force of the front arm's walking-wheel (S_F_QB_GunLun) on the downhill section (from $10 \mathrm{~s}$ to $85 \mathrm{~s}$ ); however, the result is opposite on the uphill section (from $85 \mathrm{~s}$ to $158 \mathrm{~s}$ ). The reason is that the center of mass of the robot moves a little to the walkingwheel whose position is higher due to the line slope. However, the maximum difference between two walkingwheels' forces is about $20 \mathrm{~N}$. There are some points that the forces of two walking-wheels are nearly equal, such as $12.5 \mathrm{~s}$, $42.5 \mathrm{~s}, 127.5 \mathrm{~s}$, and $155.5 \mathrm{~s}$, because the robot's acceleration reaches the maximum.

From $85 \mathrm{~s}$ to $125 \mathrm{~s}$ (uniform speed walking) and from $130 \mathrm{~s}$ to $135 \mathrm{~s}$ (stop state) and from $135 \mathrm{~s}$ to $140 \mathrm{~s}$ (accelerated walking), the force of the front arm's walking-wheel $\left(F_{F}\right.$ shown in Figures 4 and 5) is larger than the force of the back arm's walking-wheel ( $F_{B}$ shown in Figures 4 and 5$)$, which is consistent with the theoretical analysis of formulas (3) and (11).
From $125 \mathrm{~s}$ to $130 \mathrm{~s}$ (decelerated walking), the forces of two arms' walking-wheel $\left(F_{F}\right.$ and $F_{B}$ shown in Figure 6$)$ are changing with the acceleration of robot ( $a$ shown in Figure 6), which is consistent with the theoretical analysis of formulas (17).

When the robot walks at the uniform speed, the sum force of two walking-wheels (ADD (F_QB $+F_{-}$HB) ) almost stays the same, which is about equal to the robot weight, but the sum force is affected by robot's acceleration fluctuating slightly.

4.2.4. Torque Curves of Two Walking-Wheels. Figure 10 shows the driving torque curves of two walking-wheels. It can be seen that two walking-wheels provide the braking torque on downhill section and the driving torque on uphill section, and the torques of two walking-wheels increase with the increase of the line slope, which is consistent with the theoretical analysis of formulas (6), (8), (14), and (23).

The driving torques of two walking-wheels are the power source of the robot, which determines the walking characteristics of the robot. Taking robot walking on the uphill section as an example, the changing processes of two walking-wheels' torques are analyzed when the robot is at the walking state of acceleration, uniform speed, deceleration, stopping, and the downhill section is the opposite.

From $130 \mathrm{~s}$ to $135 \mathrm{~s}$, the robot stops on the uphill section, and the walking-wheels do not rotate. It is necessary that the walking-wheels provide the driving torque to avoid the robot skidding, and the walking-wheel torques keep unchanging.

From $135 \mathrm{~s}$ to $140 \mathrm{~s}$, the robot accelerates on the uphill section; the walking-wheels provide the upward acceleration. The acceleration increases gradually from zero to the maximum and then decreases to zero. Therefore, the walking-wheel torques increase at first and then decrease.

From $140 \mathrm{~s}$ to $153 \mathrm{~s}$, the robot walks at a uniform speed on the uphill section; the walking-wheel torques increase as the line slope increases gradually. Due to the small fluctuation of robot box's acceleration (shown in Figure 8), the walking-wheel torques also fluctuate slightly.

From $153 \mathrm{~s}$ to $158 \mathrm{~s}$, the robot decelerates on the uphill section; the robot's gravity provides the downward acceleration. The acceleration increases gradually from zero to the maximum and then decreases to zero. Therefore, the walking-wheel torques decrease at first and then increase.

4.2.5. Distance Curve of Two Walking-Wheels. Figure 11 shows the variation curve of the distance of two walkingwheels, the maximum value is about $698 \mathrm{~mm}$, the minimum value is about $691 \mathrm{~mm}$, and the fluctuation range is very small, which indicates that two walking-wheels walk smoothly and do not easily skid.

4.2.6. Analysis of the Simulation Results. The above analysis shows that the robot's walking process on the catenary transmission line is stable.

When the robot walks along the downhill, the walkingwheels provide braking torque because the robot's gravity 


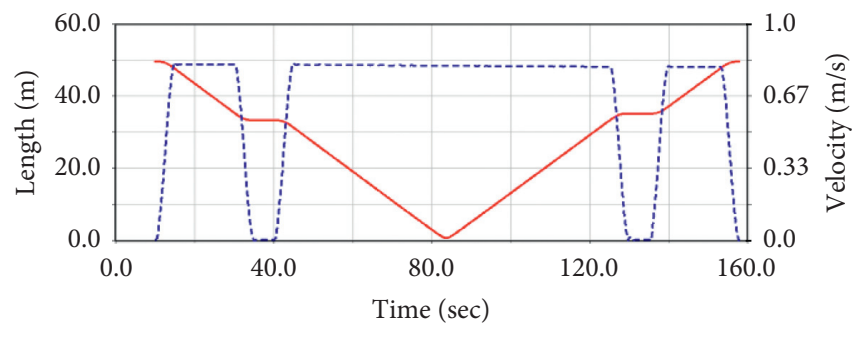

$$
\text { - S_DM_Box }
$$

Figure 7: Velocity and displacement curves of box.

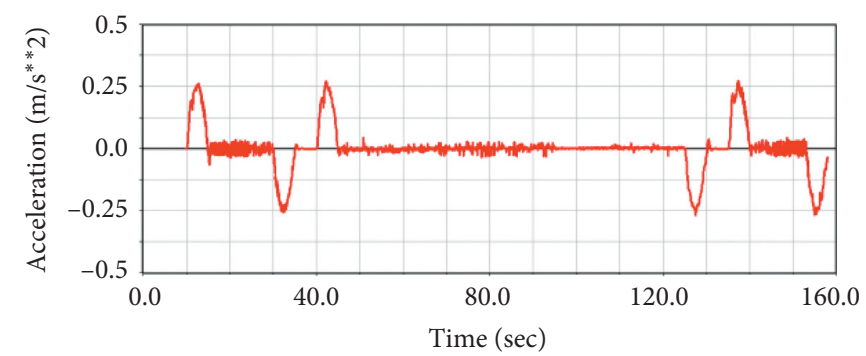

— Box.CM_Acceleration

Figure 8: Acceleration curve of box.

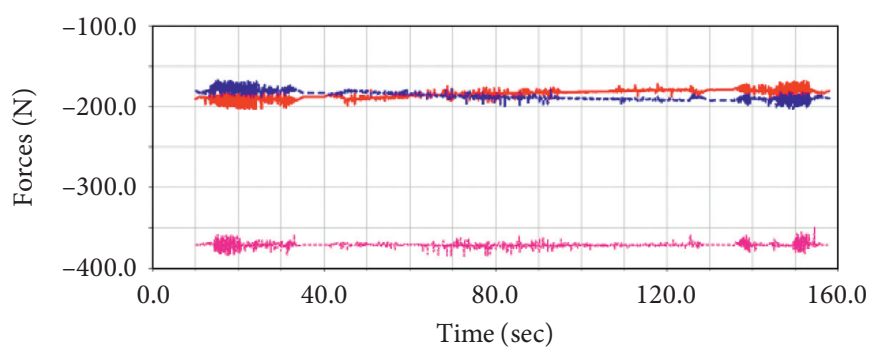

- S_F_QB_GunLun

-.- S_F_HB_Gunlun

- - ADD (F_QB + F_HB)

Figure 9: Force curves of two walking-wheels.

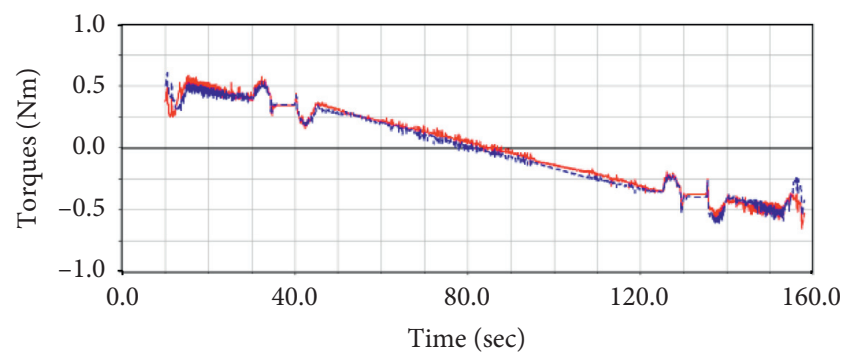

- S_T_HB_GunLun

-.- S_T_QB_GunLun

FIGURE 10: Torque curves of walking-wheels.

does the positive work. However, when robot walks along the uphill, the walking-wheels provide the driving torque because the robot's gravity does negative work.

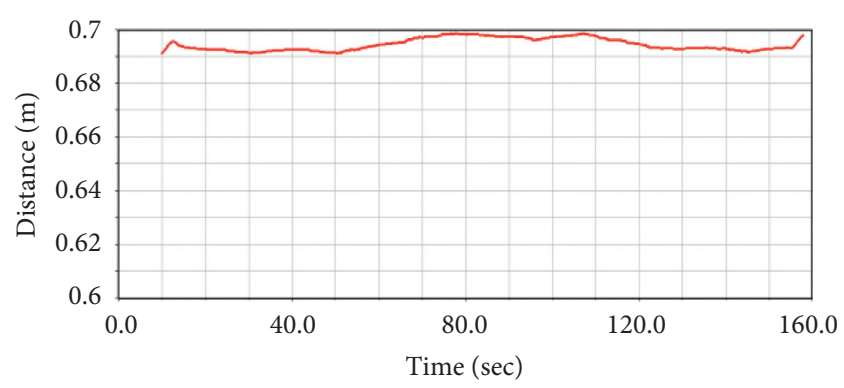

FIGURE 11: Distance curve of two walking-wheels.

Because the forces of two walking-wheels are nearly balanced and two walking-wheels contact the line very well, the good line adaptability of the robot is guaranteed and the climbing ability of the robot is improved. 


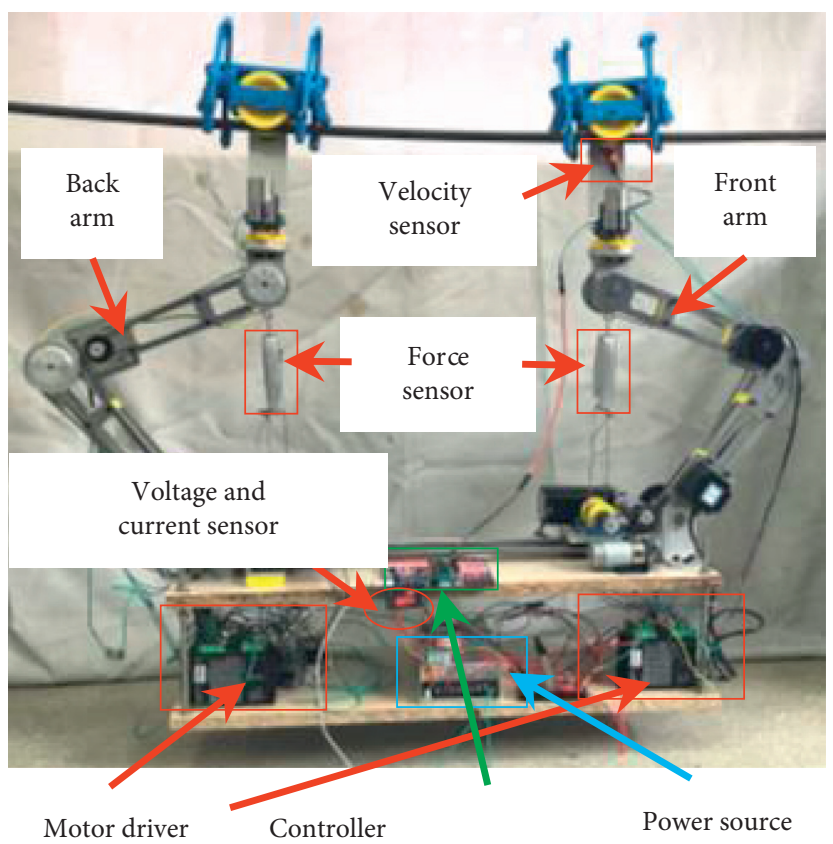

Figure 12: Prototype robot system.

In addition, when the robot walks along the line, each joint of the arm is relaxed, the distance of two walkingwheels does not fluctuate greatly, and the movement of the box is stable. Therefore, the robot box can always stay parallel with the line to ensure that the relationship between the detection equipment and the line keeps unchanging, which is beneficial for the robot to carry out the inspection operation.

\section{Prototype Experiment of the Robot Walking along the Transmission Line}

In order to verify the walking characteristics of the dual-arm inspection robot with flexible-cable, the prototype robot was made, and the forces of two flexible-cables and the torques of the walking-wheel were measured under the different line slopes. The weight of the prototype robot is about $26 \mathrm{~kg}$ and the transmission line slope is from $0^{\circ}$ to $15^{\circ}$.

5.1. Prototype Robot System. The prototype robot system is shown in Figure 12; besides the motor which drives the robot to move, the control and test system includes controller, motor driver, power source, force sensor, velocity sensor, voltage and current sensor, etc..

5.2. Experiment of the Prototype Walking along the Transmission Line. In the laboratory, the transmission line for experiment was set at four-kind slope of $0^{\circ}, 5^{\circ}, 10^{\circ}$, and $15^{\circ}$. As shown in Figure 13, the robot walked along the uphill line at each slope.
5.2.1. Forces of the Flexible-Cables. It is difficult to measure the forces between two walking-wheels and the line directly, but it can be reflected by measuring the forces of two flexible-cables, because two flexible-cables bear most of the weight of robot and their forces are consistent with the forces of two walking-wheels. The forces of two flexible-cables were measured by the force sensors, as shown in Figure 14 .

Figure 14 shows that the force of the flexible-cable of the front arm increases with the increase of the line slope, and the force of the flexible-cable of the back arm is opposite; however, the sum force of two flexible-cables almost stays the same, which is consistent with the simulation result of the Figure 9.

5.2.2. Torque of the Walking-Wheel. The voltage and current of the motor which drove the walking-wheel were measured by the voltage and current sensor, and the velocity of walking-wheel was measured by the velocity sensor. The torque of the walking-wheel could be derived by

$$
M=\frac{U I \eta}{\omega},
$$

where $U$ and $I$ are the voltage and current of the motor, $\eta$ is the transmission efficiency from the motor to the walkingwheel, and $\omega$ is the angular velocity of the walking-wheel.

So, when the robot walked along the different line slope, the torque of walking-wheel could be obtained by the control and test system, as shown in Figure 15.

Figure 15 shows that the driving torque increases with the increase of the line slope, which is consistent with the simulation result of Figure 10. However, the torque value is 


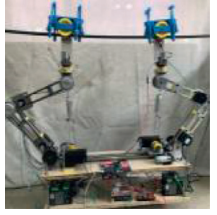

(a)

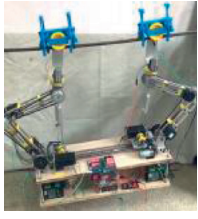

(b)

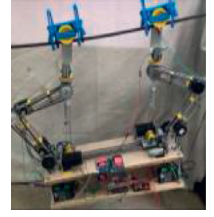

(c)

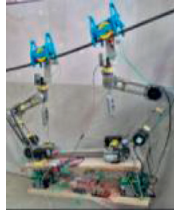

(d)

FIGURE 13: Experiment of robot walking along the line. (a) $0^{\circ}$ line. (b) $5^{\circ}$ line. (c) $10^{\circ}$ line. (d) $15^{\circ}$ line.

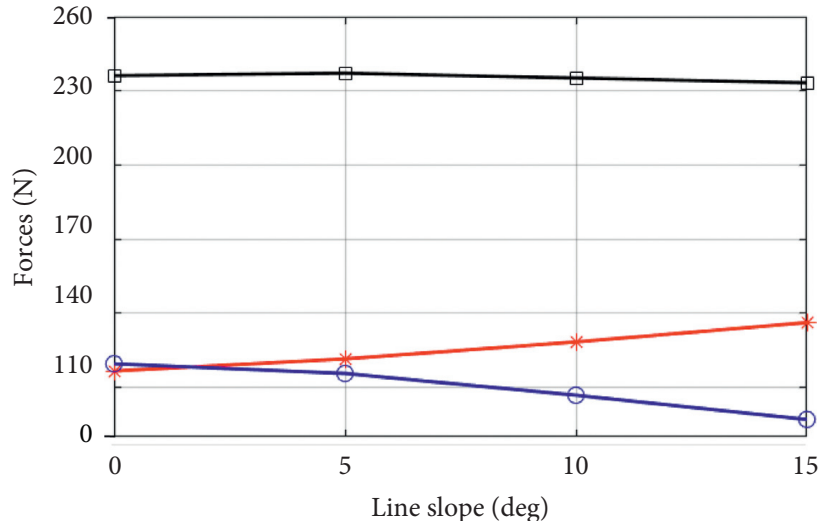

$\square \quad$ Experimental data of the sum force of two arms

- Fitting curve of the sum force of two arms

* Experimental data of the force of the front arm

- Fitting curve of the force of the front arm

O Experimental data of the force of the back arm

Fitting curve of the force of the back arm

Figure 14: Forces of the flexible-cables.

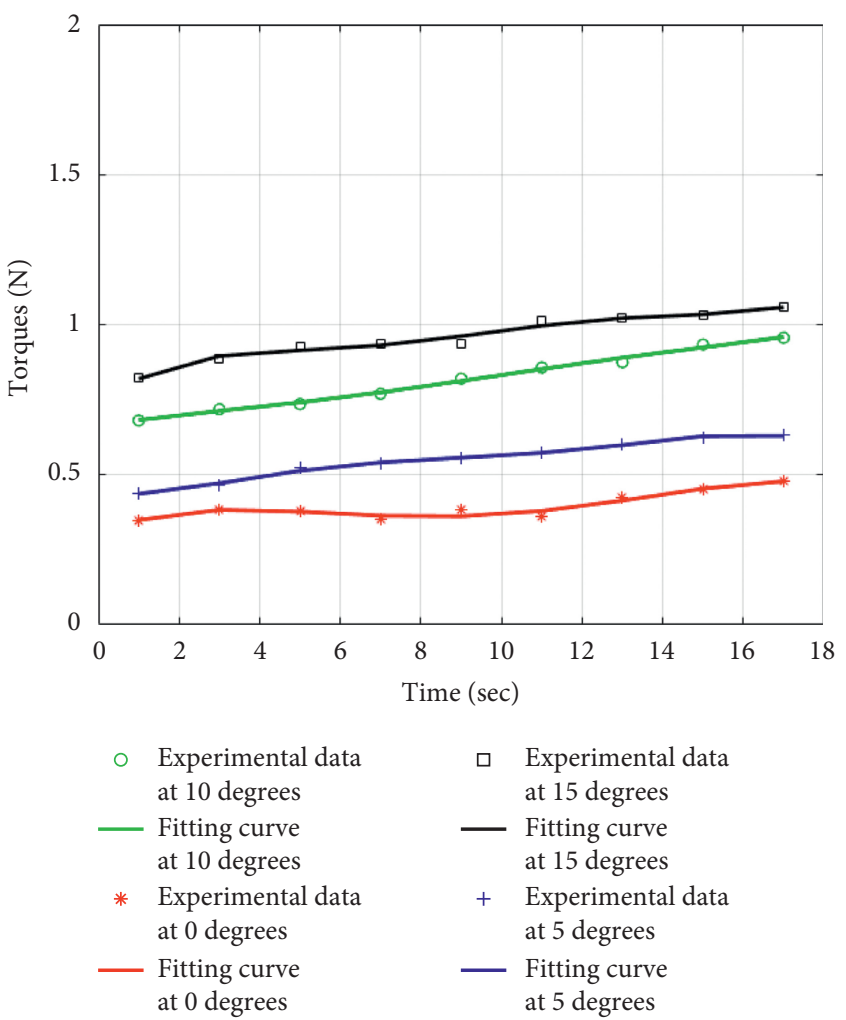

Figure 15: Torques of the front walking-wheel. slightly larger than the simulation result; for example, the torque of the $5^{\circ}$ line slope; it is mainly because the frictional resistances of many moving parts were not considered in the simulation. The fluctuation of the torque is affected by the deformation of the line.

\section{Conclusion}

Because the overhead transmission line has a catenary shape, the robot is always walking uphill or downhill during the inspection process, which has an obvious influence on the dynamic characteristics of the robot walking.

The dynamic characteristics of the robot walking at various states are studied, through theoretical analysis, simulation experiment, and prototype experiment; the following conclusions are obtained:

(1) Compared with other similar inspection robots, the structure of the dual-arm inspection robot with flexible-cable is unique, and the walking-posture of the robot can change with the line slope because of each joint of the arm being relaxed.

(2) Because the walking-posture of the robot can change, the center of gravity of the robot is always between two walking-wheels, and the forces and torques of two walking-wheels are almost equal which enables the robot to have good line adaptability and climbing ability. 
(3) The walking-wheel needs to provide the driving torque to overcome the robot gravity on the uphill section, and the walking-wheel needs to provide the braking torque to avoid robot runaway on the downhill section.

(4) The force and torque of the walking-wheel are mainly affected by the weight of the robot, the line slope, and the acceleration of the robot walking. The weight of the robot is constant and the acceleration of the robot walking is generally small, so the line slope is the main affecting factor.

(5) The fluctuation of two walking-wheels' distance is very small, and the velocity curve of the center of mass of the box is smooth, which indicates that the robot walks very stably.

All the works verified that the structure of the robot is rational and advantageous. Future work will focus on developing the navigation control system of the robot, so that the robot can walk along the transmission line stably to complete the inspection task.

\section{Data Availability}

The data used to support the findings of this study have been deposited in the [Research on Dual-Arms Inspection Robots Walking along Transmission Line] repository ([DOI: 10. 15918/j.tbit1001-0645.2019.08.007]), the [Analysis for Crossing Obstacle Distance of Dual-Arms Inspection Robot] repository ([DOI: 10.12068/j.issn.1005-3026.2017.09.017]), and the [Design of a Novel Dual-Arm Inspection Robot with Flexible Cable Robot] repository ([DOI: 10.3724/sp.j.1218. 2013.00319])

\section{Conflicts of Interest}

The authors declare that they have no conflicts of interest.

\section{Acknowledgments}

This study was supported by Basic Scientific Research Project of the Educational Department of Liaoning Province (Grant no. LJ2020JCL024), Nature Fund Project of Liaoning Province (Grant No. 2019-ZD-0047), and Project Supported by Discipline Innovation Team of Liaoning Technical University (Grant nos. LNTU20TD-28).

\section{References}

[1] P. Debenest, M. Guarnieri, K. Takita et al., "Expliner-robot for inspection of transmission lines," in Proceedings of the EEE International Conference on Robotics and Automation Pasadena, pp. 3978-3984, CA, USA, May 2008.

[2] P. Debenest and M. Guarnieri, Expliner-From Prototype towards a Practical Robot for Inspection of High-Voltage Lines Applied Robotics for the Power Industry, pp. 1-6, IEEE, Montréal, Canada, 2010.

[3] N. Pouliot and S. Montambault, "Field-oriented developments for LineScout Technology and its deployment on large water crossing transmission lines," Journal of Field Robotics, vol. 29, no. 1, pp. 25-46, 2012.
[4] N. Pouliot, P.-L. Richard, and S. Montambault, "LineScout technology opens the way to robotic inspection and maintenance of high-voltage power lines," IEEE Power and Energy Technology Systems Journal, vol. 2, no. 1, pp. 1-11, 2015.

[5] A. Fonseca, R. Abdo, and J. O. Alberto, "Robot for inspection of transmission lines," in Proceedings of the 2nd International Conference on Applied Robotics for the Power Industry, ETH Zurich, Switzerland, September 2012.

[6] T. Lorimer and E. D. Boje, "A simple robot manipulator able to negotiate power line hardware," in Proceedings of the 2nd International Conference on Applied Robotics for the Power Industry, pp. 136-141, ETH Zurich, Switzerland, September 2012.

[7] R. Timothy and E. D. Boje, "Obstacle avoidance for a power line inspection robot," in Proceedings of the 2nd International Conference on Applied Robotics for the Power Industry, ETH Zurich, Switzerland, September 2012.

[8] L. Wang, S. Cheng, and J. Zhang, "Development of a LineWalking Mechanism for Power Transmission Line Inspection Purpose," in Proceedings of the the 2009 IEEE/RSJ International Conference on Intelligent Robots and Systems, St. Louis, USA, October 2009.

[9] L. Wang, F. Liu, Z. Wang et al., "Development of a Novel power transmission line inspection robot," in Proceedings of the 2010 1st International Conference on Applied Robotics for the Power Industry Delta Centre-Ville, Montréal, Canada, October 2010.

[10] X. Zhu, H. Wang, L. Fang et al., "Analysis of drive dynamic performance and position-pose of autonomous robot for transmission line inspection," Chinese Journal of Mechanical Engineering, vol. 42, no. 12, pp. 143-150, 2006.

[11] X. Zhu, J. Zhou, H. Wang et al., "Experiments and mechanism of obstacle negotiation of an inspection robot for transmission lines," Chinese Journal of Mechanical Engineering, vol. 45, no. 2, pp. 119-125, 2009.

[12] G. Wu, H. Cao, P. I. Yuan et al., "An autonomous inspection robot for high voltage bundled transmission line and its application," Engineering Journal of Wuhan University, vol. 45, no. 1, pp. 96-102, 2012.

[13] H. Cao, G. Wu, Z. H. E. N. Tuo et al., "Obstacle detection and locating method of an autonomous inspection robot for high voltage transmission line," Engineering Journal of Wuhan University, vol. 45, no. 2, pp. 229-234, 2012.

[14] T.-Y. Zhang, Z. H. A. N. G. Guo-xian, and J. Jin, "Dynamics modeling and analysis for power lines inspection robot," Journal of System Simulation, vol. 20, no. 18, pp. 4982-4986, 2008.

[15] W.-L. Zhang, Z. G. Xian, L. H. Bin et al., "Structure design and kinematics analysis of a new inspection robot," Journal of Machine Design, vol. 27, no. 12, pp. 50-52, 2010.

[16] M. H. Korayem, H. Tourajizadeh, and M. Bamdad, "Dynamic load carrying capacity of flexible cable suspended robot: robust feedback linearization control approach," Journal of Intelligent \& Robotic Systems, vol. 60, no. 3-4, pp. 341-363, 2010.

[17] M. H. Korayem and H. Tourajizadeh, "Maximum DLCC of spatial cable robot for a predefined trajectory within the workspace using closed loop optimal control approach," Journal of Intelligent \& Robotic Systems, vol. 63, no. 1, pp. 75-99, 2011.

[18] L. Fang, Y. Wei, and G. Tao, "Design of a Novel dual-arm inspection robot with flexible cable," Robot, vol. 35, no. 3, pp. 319-325, 2013.

[19] Y. Wei and L. Fang, "Kinematical modeling and simulation of dual-arms inspection robot with flexible-cable," Journal of 
Liaoning Technical University (Natural Science), vol. 37, no. 2, pp. 389-395, 2018.

[20] Y. Wei, L. Fang, and G. Tao, "Analysis for crossing obstacle distance of dual-arms inspection robot," Journal of Northeastern University (Natural Science), vol. 38, no. 9, pp. 1293-1297, 2017.

[21] Y. Wei and L. Fang, "Research on dual-arms inspection robots walking along transmission line," Transactions of Beijing Institute of Technology, vol. 39, no. 8, pp. 813-818, 2019.

[22] W. Jiang, Z.-Y. Zhou, W. Chen et al., "Manipulator double close loop autonomous localization control of high-voltage cable mobile operation robot," Transactions of Beijing Institute of Technology, vol. 39, no. 6, pp. 589-596, 2019.

[23] W. Jiang, G. Zuo, D. H. Zou, H. Li, J. J. Yan, and G. C. Ye, "Autonomous behavior intelligence control of self-evolution mobile robot for high-voltage transmission line in complex smart grid," Complexity, vol. 2020, Article ID 8843178, 17 pages, 2020.

[24] P. Božek, M. Aiman Al Akkad, B. Peter et al., "Navigation control and stability investigation of a mobile robot based on a hexacopter equipped with an integrated manipulator," International Journal of Advanced Robotic Systems, vol. 14, pp. 1-13, 2017.

[25] R. Pirník, M. Hruboš, D. Nemec et al., "Integration of inertial sensor data into control of the mobile platform," Federated Conference on Software Development and Object Technologies, vol. 511, pp. 271-282, 2015. 